\title{
DNS INVESTIGATION INTO THE EFFECT OF FREE-STREAM TURBULENCE ON HAIRPIN-VORTEX EVOLUTION
}

\author{
KAZUO MATSUURA \\ Graduate School of Science and Engineering, Ehime University, Japan
}

\begin{abstract}
The effects of free-stream turbulence (FST) on the evolution of hairpin vortices during the process of laminar-turbulent transition are investigated by direct numerical simulation. The simulations are conducted for FST intensities of $0-6 \%$ and a free-stream Mach number of 0.5 . FST changes the symmetry of the hairpin vortex, the arrangement of vortices inside the boundary layer, the location of high friction at the wall, and the scales of the vortices. The relationship between high-friction regions at the wall and hairpin vortices convected downstream is investigated by analyzing the computed flow fields. When FST is introduced along with inlet sinuous disturbances, asymmetric hairpin vortices and numerous secondary hairpin vortices, which are different from the case of no FST, are generated. The boundary-layer transition is characterized by an increase in skin-friction. As a result of vortex creation very close to the wall, high-friction regions are generated. To detect the vortex parts responsible for generating the high-friction regions, a new judgement algorithm for interior points is derived based on the Euler angle. The interior points of the near-wall vortices responsible for generating such highfriction regions are successfully visualized as a group of points in $\mathbf{R}^{3}$. The high-friction regions are the tips of hairpin legs and the regions of near-wall vortices induced beneath a hairpin packet. These regions often correspond to the periphery of the hairpin packets.

Keywords: hairpin vortex, boundary layer, laminar-turbulent transition, turbulence, direct numerical simulation, stability.
\end{abstract}

\section{INTRODUCTION}

Hairpin vortices are considered to be elementary building blocks of turbulence near a solid wall [1]. Since the work of Theodorsen [2], there have been many studies on hairpin/horseshoe vortices, and the generation of hairpin vortices has been discussed in numerous reports [3]-[8]. Among previous studies, Moin et al. [9] numerically examined the deformation of a hairpin-shaped vortex filament under self-induction and in the presence of shear using the Biot-Savart law and reported a mechanism of ring vortex generation in turbulent shear flows. Acarlar and Smith [10] experimentally visualized the dynamics of hairpin vortices in the downstream wake of a hemispheric obstacle. Hon and Walker [11] proposed a numerical method based on a Lagrangian vortex method that allows accurate computations of the trajectory of a three-dimensional vortex having a small core radius. Using this method, they clarified that a two-dimensional vortex containing small threedimensional disturbances distorts into a complex shape, with subsidiary hairpin vortices forming outboard of the original hairpin vortex. Singer and Joslin [12] computationally studied the formation and growth of a hairpin vortex in a flat-plate boundary layer and its later development into a young turbulent spot. The initial hairpin vortex was triggered by fluid injection through a slit in the wall. They reported the development of multiple hairpin vortex heads between stretched legs, the formation of new vortices beneath the streamwiseelongated vortex legs, and the formation of a traveling region of highly disturbed flow with an arrowhead shape, similar to that of a turbulent spot. By direct numerical simulation (DNS), Zhou et al. [13], [14] studied the evolution of a symmetric pair of quasistreamwise vortical structures extracted from the two-point correlation tensor of turbulent channel flow data by 
a linear stochastic estimation procedure. They observed that sufficiently strong hairpin vortices generate a hierarchy of secondary hairpin vortices, and the mechanism of their creation closely resembles the formation of the primary hairpin vortex. Using a compressible DNS, Liu and Chen [15] studied the nonlinear stages of laminar-turbulent transition. They discussed the coherent vortex structure appearing in the late stages of transition and the formation mechanism of single vortex rings, multiple vortex rings, and small length scales. At the inflow, they assumed two-dimensional waves and a pair of oblique waves, in addition to the laminar boundary layer profile, to reproduce the transition of the K-regime. Duguet et al. [16] studied the region in phase space separating transitional from relaminarizing trajectories for the Blasius boundary layer and found a quasicyclic mechanism for the generation of hairpin vortex offspring. Cohen et al. [17] proposed a model consisting of minimal flow elements that evolves into packets of hairpins. The three components of the model are simple shear, a counter-rotating vortex pair having finite streamwise vorticity magnitude, and a two-dimensional wavy (in the streamwise direction) spanwise vortex sheet. Eitel-Amor et al. [18] studied the characteristics of hairpin vortices in turbulent boundary layers using parallel and spatially developing simulations. They found that secondary hairpins are only created shortly after initialization, with all rotational structures decaying at later times. They also reported that the regeneration process is rather short-lived and may not be sustained once a turbulent background has developed. Sabatino and Maharjan [19] conducted an experimental study of hairpin vortex formation in a laminar boundary layer by fluid injection through a narrow slot. They discussed the hairpin vortex head, legs, and secondary hairpin vortex, focusing on its circulation strength. Kim et al. [20] studied the effects of background noise on the generation of coherent packets of hairpin vortices. They showed that the hairpins become asymmetric, leading to more complicated packet structures than are observed in the symmetric hairpin vortex train of the flow with a clean background. They also examined the robustness of the autogeneration mechanism by comparing the rate of growth in the vertical and spanwise dimensions, as well as the distance between successive vortices, in chaotic packets with those in clean packets.

Although there have been many studies on hairpin vortices, investigations into the effects of free-stream turbulence (FST) on the evolution of hairpin vortices are relatively scarce. Additionally, to the best of our knowledge, the effects of FST on the relationship between skin-friction at the wall and hairpin vortices are not well understood. Therefore, this study investigates these effects for the scenario of laminar-turbulent transition on a flat plate.

In Section 2, the computational methods employed in this work are described. In Section 3 , the various computational cases are explained. Section 4 introduces the newly devised algorithm for judging interior points of vortices, before Section 5 discusses the effects of FST on the evolution of hairpin vortices, focusing on the relationship between the high-friction regions at the wall and hairpin vortices. Finally, in Section 6, the conclusions to this study are presented.

\section{COMPUTATIONAL METHODS}

The governing equations are the unsteady three-dimensional fully compressible NavierStokes equations written in general coordinates for body-fitted mesh geometries. The system of equations is closed by the perfect gas law. A constant Prandtl number of $P r=0.72$ is assumed. The equations are solved using a sixth-order finite-difference method. Timedependent solutions to the governing equations are obtained using the third-order explicit Runge-Kutta scheme. The present numerical method has been extensively validated for the prediction of transitional and turbulent subsonic flows [21], [22]. 
Regarding the boundary condition, inflow profiles are specified at the inlet. Depending on the computational case, disturbances are added to a base inflow profile. The base inflow profile is obtained by solving the boundary layer equation [23]. At the outflow and upper boundaries, non-reflecting boundary conditions with a mean static pressure of $p_{\infty}=101,325$ Pa are imposed. At the wall, the non-slip, isothermal wall condition $T_{\infty}=273.15 \mathrm{~K}$ is imposed. Periodicity is imposed in the spanwise direction. The computational domain is a rectangular region with dimensions $416.31 \delta_{\text {in }}, 80.0 \delta_{\text {in }}$ and $22.0 \delta_{\text {in }}$ in the $x$ (streamwise), $y$ (wall-normal) and $z$ (spanwise) directions, respectively for Cases A, C and D, and $318.0 \delta_{\text {in }}, 80.0 \delta_{\text {in }}$ and $22.0 \delta_{\text {in }}$ for Case B. Here, $\delta_{\text {in }}$ is the displacement thickness at a virtual point $\mathrm{x}=300.79 \delta_{\text {in }}$. The Reynolds number based on the free-stream quantity and the length $\delta_{\text {in }}$ is 1000 . The inlet position of the computational domain for Cases A, C and D is $\mathrm{x}=300.79 \delta_{\text {in }}$, and that for Case

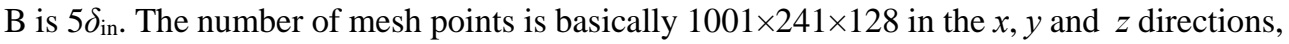
respectively, for Cases A. C and D, and $765 \times 241 \times 128$ for Case B.

\section{COMPUTATIONAL CASES}

Four cases (A-D) are considered in this study. The scenario for each case is summarized in Table 1. Cases A and B are reference examples. Case A has no FST, and the transition of the K-regime is reproduced. Case B has FST with a high intensity, Tu $=6 \%$, and produces a bypass transition. In Cases $\mathrm{C}$ and $\mathrm{D}$, the evolution of hairpin vortices is influenced by FST. The FST has an intensity of $0.5 \%$ in Case C, $1 \%$ in Case D. The mean free-stream Mach number is 0.5 in all cases. In Case A, disturbances comprising a two-dimensional TollmienSchlichting wave and a pair of oblique waves are superimposed on the Blasius solution [15]. In Cases B-D, FST is superimposed on the free-stream region of the velocity profile. In Cases $\mathrm{C}$ and $\mathrm{D}$, the initial conditions are the resultant instantaneous flow field of Case A. At the inlet, the sinuous disturbances from Case A and isotropic FST are added to the inflow laminar boundary layer profile.

The FST added to Cases B-D must reproduce the structures of real turbulence to some extent. In the present study, FST is obtained from an instantaneous flow field of isotropic turbulence that had been computed separately. To introduce FST to the computational domain, velocity fluctuations in planes with a constant separation distance are extracted from the instantaneous flow field. After an appropriate scaling of the turbulence intensities, the resultant velocity perturbations are specified at the inlet boundary at every time step. Details of generating FST are explained in [21].

\section{ALGORITHM FOR JUDGING INTERIOR POINTS OF A VORTEX}

Generally, in transitional boundary layers, numerous vortices are generated depending on the distance from the wall. In addition, free-stream eddies exist in the present flow fields in Cases $\mathrm{C}$ and $\mathrm{D}$. Because it is difficult to directly track the vortices and define their region, a method of dimensional reduction is derived and applied to the present flow fields to extract and

Table 1: Computational cases. IC: Initial condition; LBL: Laminar boundary layer; BC: Boundary condition; SD: Sinuous disturbances; IST: Isotropic turbulence.

\begin{tabular}{|c|c|c|c|}
\hline Case & FST intensity & IC & Inflow BC \\
\hline A & $0 \%$ & Steady LBL profile & LBL+SD \\
\hline B & $6 \%$ & Steady LBL profile & LBL+FST(IST) \\
\hline C & $0.5 \%$ & \multirow{2}{*}{ Case A } & LBL+SD+FST(IST) \\
\hline D & $1 \%$ & & \\
\hline
\end{tabular}


understand the dynamics of the hairpin and its associated vortices. In this method, the interior region of a vortex tube is expressed using particles extracted by an algorithm devised in this study. This algorithm extracts mesh points surrounded by the iso-surface of a function $\mathrm{f}(x, y, z)$ $=$ const. Although an arbitrary function could be used for $\mathrm{f}(x, y, z)$, the second invariance of the velocity gradient tensor is employed in this study.

The coordinate system for the proposed algorithm is shown in Fig. 1. First, it is determined whether a mesh point $\mathbf{P}_{0}$ is an interior point. Around $\mathbf{P}_{0}$, a unit circle $C$ is considered in $\mathbf{R}^{3}$. On the circle, equispaced points $\mathrm{PC}_{\mathrm{k}}$ are generated for $\mathrm{k}=1, \ldots, \mathrm{N}$. Here, the circle $\mathrm{C}$ around $\mathbf{P}_{0}$ is generated by rotating a unit circle $\mathrm{C}_{0},(x, y, \mathrm{z})=(\cos \varphi, \sin \varphi, 0)^{\mathrm{T}}$, in $\mathbf{R}^{3}$. Using the Euler angle, this transformation is expressed as follows:

$$
\begin{gathered}
\left(\begin{array}{l}
x \\
y \\
z
\end{array}\right)=\mathrm{T}(\alpha, \beta, \gamma)\left(\begin{array}{c}
\cos \phi \\
\sin \phi \\
0
\end{array}\right), \\
\mathrm{T}(\alpha, \beta, \gamma)= \\
\left(\begin{array}{ccc}
\cos \gamma \cos \beta \cos \alpha-\sin \gamma \sin \alpha & \cos \gamma \cos \beta \sin \alpha+\sin \gamma \cos \alpha & -\cos \gamma \sin \beta \\
-\sin \gamma \cos \beta \cos \alpha-\cos \gamma \sin \alpha & -\sin \gamma \cos \beta \sin \alpha+\cos \gamma \cos \alpha & \sin \gamma \sin \beta \\
\sin \beta \cos \alpha & \sin \beta \sin \alpha & \cos \beta
\end{array}\right)
\end{gathered}
$$

From the center $\mathbf{P}_{0}$ to each point $\mathrm{PC}_{\mathrm{k}}$, a line segment $\mathrm{R}_{\mathrm{k}}$ is drawn. On each line segment, equispaced points $\mathrm{PR}_{\mathrm{k}, \mathrm{m}}$ are generated for $\mathrm{m}=1, \ldots$, M. From an ordered set of the values of $\mathrm{f}\left(\mathrm{PR}_{\mathrm{k}, \mathrm{m}}\right)$ obtained by linear interpolation, the existence of the iso-surface of $\mathrm{f}(x, y, z)=\varepsilon$ on the line segment can be judged, i.e. the cutting of the line segment by the iso-surface. Here, $\varepsilon$ is a threshold value used to visualize the vortices. If the iso-surface of $\mathrm{f}(x, y, z)=\varepsilon$ exists on all

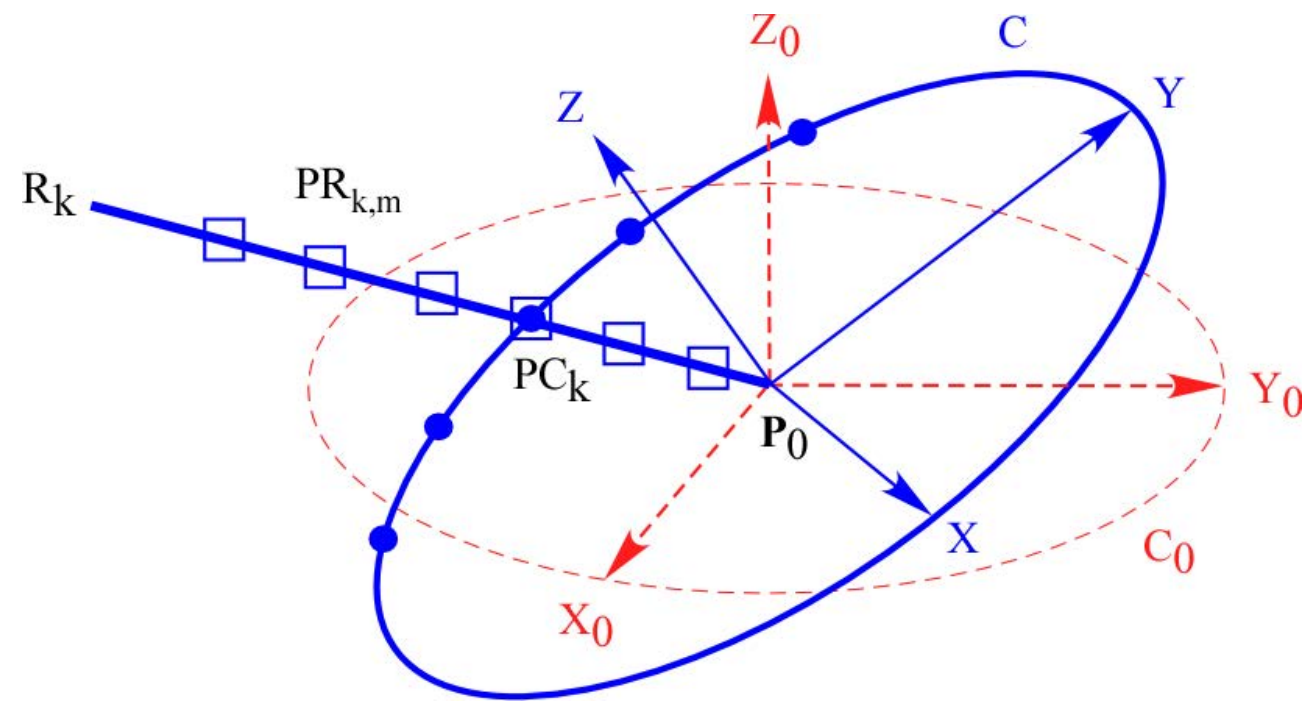

Figure 1: Coordinate system for judging interior points of vortices surrounded by an isosurface. 


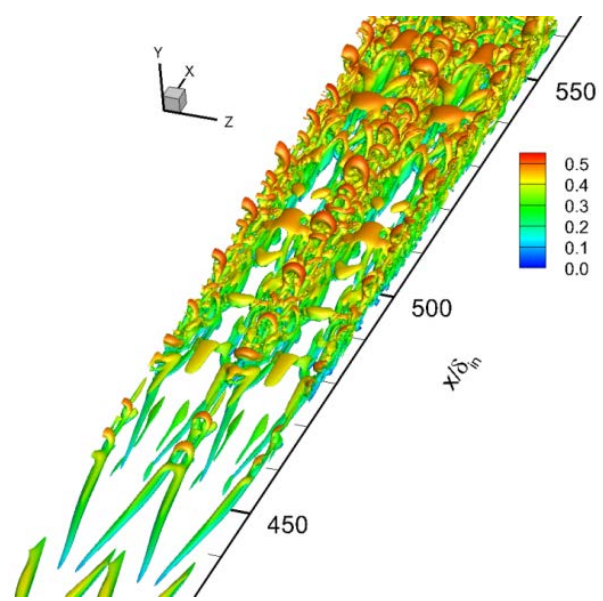

Case A

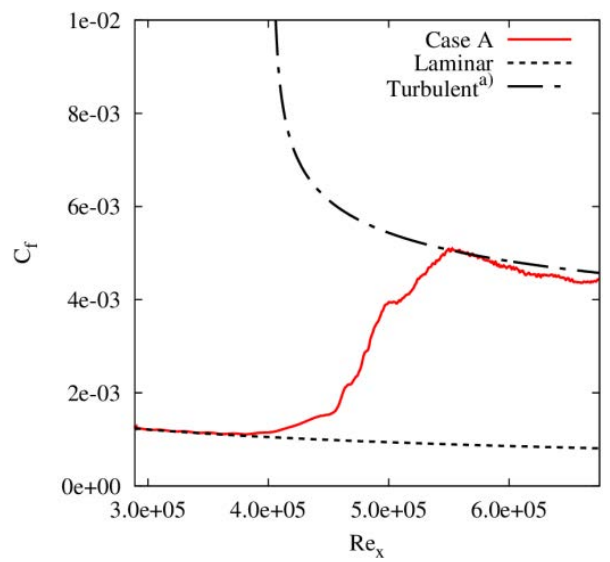

Case A

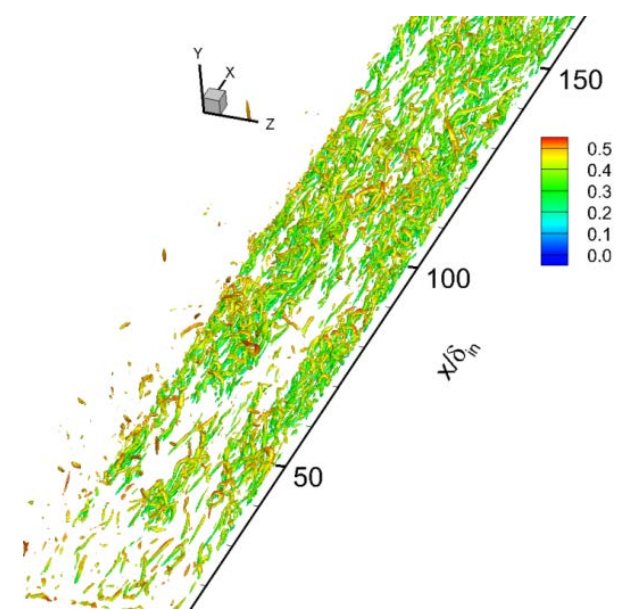

Case B

(a)

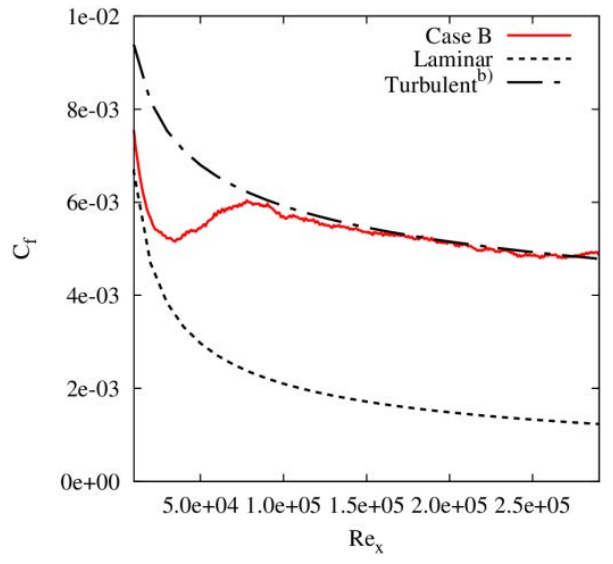

Case B

(b)

Figure 2: Vortex structures that appear during laminar-turbulent transition and associated skin-friction coefficients along streamwise Reynolds number in Cases A and B [29]. (a) Instantaneous visualization of vortices created in the process of laminarturbulent transition. Vortical structures are visualized by the iso-surface of the second invariance of the velocity gradient tensor. The color on the iso-surface and in the legend is the Mach number, $\delta_{\text {in }}$ is the inlet displacement thickness; (b) Skin-friction coefficient along Reynolds number based on the streamwise coordinate. Superscript (a) denotes a correlation derived by Cousteix [27], and superscript (b) shows a correlation by the reference temperature method [28].

line segments $\mathrm{PR}_{k, m}, \mathrm{k}=1, \ldots, \mathrm{N}$, on the circle $\mathrm{C}$ obtained by rotating $\mathrm{C}_{0}$ at $\mathrm{P}_{0}$, then point $\mathrm{P}_{0}$ is judged to be an interior point. The length of $R_{k}$ is taken as $\delta_{\text {in }}$ in this study.

Numerous techniques have been proposed for visualizing vortices [24]. For example, Sujudi and Haimes [25] proposed an algorithm for identifying the core of a swirling flow in 
3-D discretized vector fields based on the critical point theory. Kida and Miura [26] proposed the sectional-swirl-and-pressure-minimum scheme, and individual vortices are visualized by its skeleton representation. Compared to these methods, the present method extracts flow fields within a vortex tube together with the central region, i.e. core of the vortex. This feature acts as advantageous when vortex volumes that generate high-friction regions at the wall are identified as shown in Section 5.

\section{RESULTS AND DISCUSSION}

Fig. 2 shows vortices generated in the process of laminar-turbulent transition and the skinfriction coefficient with respect to the Reynolds number based on the streamwise coordinate for Cases A and B. In Case A, symmetric hairpin vortices are generated, and the secondary hairpin vortices are well aligned. The amalgamation of hairpin vortices generated around both sides of the aligned region leads to the formation of turbulent regions. The skin-friction coefficient $\mathrm{C}_{\mathrm{f}}$ detaches from the laminar correlation at around $x=416 \delta_{\text {in }}$ and reaches the turbulent correlation at around $x=575 \delta_{\text {in }}$. In Case $\mathrm{B}$, the hairpin vortices are much smaller and more asymmetric. Dense and scarce regions are randomly generated. $C_{\mathrm{f}}$ detaches from the laminar correlation at around $\mathrm{x}=36 \delta_{\text {in }}$ and reaches the turbulent correlation at around $\mathrm{x}=81 \delta_{\text {in }}$.

As mentioned above, the initial conditions for Cases $\mathrm{C}$ and $\mathrm{D}$ are the instantaneous flow field of Case A. The computation is terminated after FST has sufficiently overtaken the convection of the hairpin vortex and penetrated into the boundary layers.

Fig. 3 shows the time evolution of hairpin vortices influenced by FST. The hairpin vortices are deformed by the FST and become asymmetric. Although the arrangement of hairpin vortices is straight in Case A, the arrangement becomes staggered in Cases C and D, and many more secondary hairpin vortices are generated. This ability to generate numerous
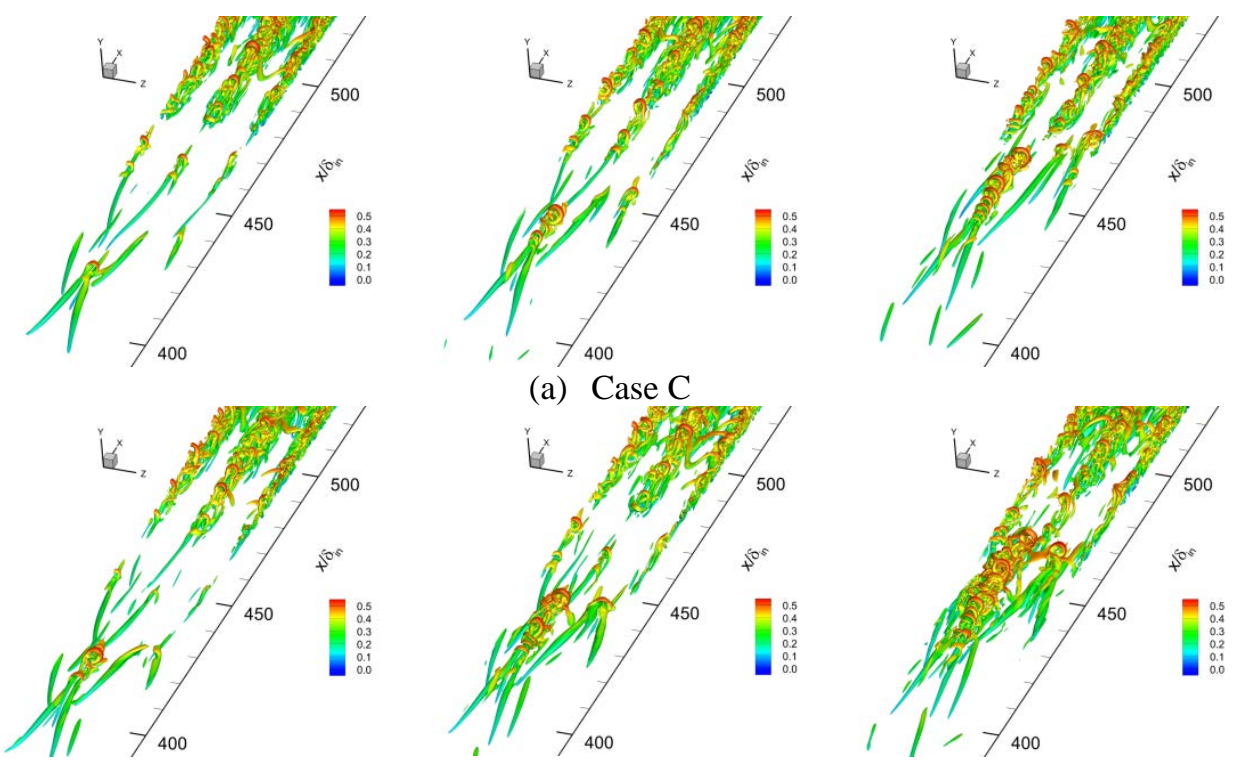

(a) Case $\mathrm{C}$
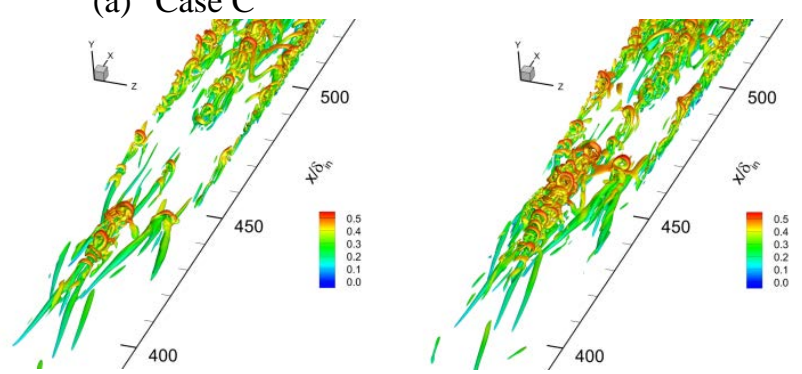

(b) Case D

Figure 3: Effect of FST on the time evolution of hairpin vortices. Left: $t^{*}=258.0 \delta_{\text {in }} / u_{\infty}$; Center: $t^{*}=281.5 \delta_{\text {in }} / u_{\infty}$; Right: $t^{*}=305.0 \delta_{\text {in }} / u_{\infty}$, The iso-surface quantity and the color on the iso-surface are the same as in Fig. 2(a). 
secondary hairpin vortices agrees with the findings reported in the author's previous paper [30].

Fig. 4 shows the relationship between the structure of the hairpin vortex packets and the high-friction regions viewed from the bottom side. High-friction regions are caused by vortices generated very close to the wall, as found from Fig. 4(a) and (b). Here, friction at the wall is defined as $\partial \mathrm{u} / \partial \mathrm{y}+\partial \mathrm{w} / \partial \mathrm{y}$ without multiplication of viscosity. In addition to the tips of hairpin legs, vortices generated beneath the primary hairpin vortex constitute the nearwall vortices, as found from Figs. 4(a) and (c).

Fig. 5 shows the time evolution of the relationship between hairpin vortices, the region of high friction, and low-speed streaks. Elongated streamwise vortices are generated near the periphery of hairpin packets as the hairpin vortices move downstream, and close to the upstream regions of the streamwise vortices. Because high friction occurs near the upstream tip of the streamwise vortices, and also beneath the primary hairpin vortex, high-friction regions are generated near the periphery of the hairpin packet. As shown in Fig. 3, the arrangements of hairpin vortices change under the influence of FST, and the regions of high friction also change accordingly.

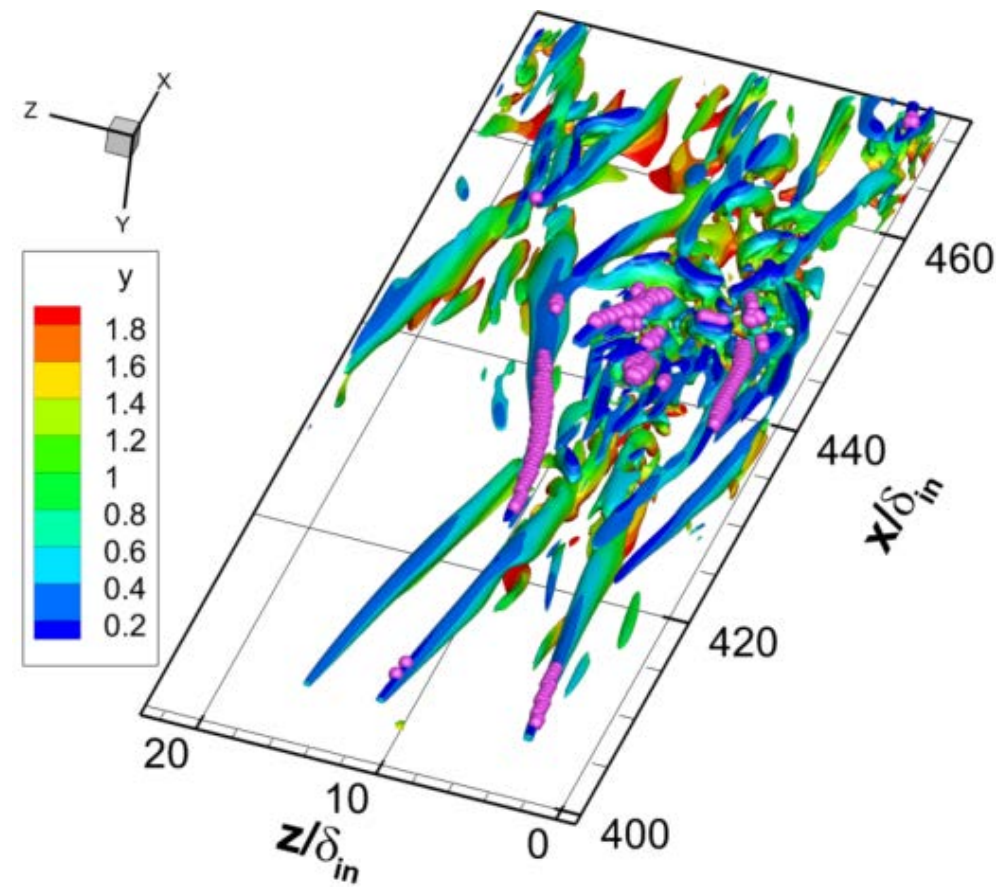

(a)

Figure 4: Relationship between the structure of hairpin vortex packets and high friction regions at $t^{*}=305.0 \delta_{\text {in }} / u_{\infty}$. (a) Vortex structures and mesh points inside the vortex structures close to high friction regions on the wall, which are viewed from the bottom side. The extracted interior points are restricted to $y<0.2 \delta_{\text {in. }}$ The colors on the iso-surface are heights from the wall, and the mesh points are shown by pink points; (b) Near-wall streamlines. The color shows the magnitude of the wall shear; (c) Streamlines passing through the cross section of the vortex structures. 


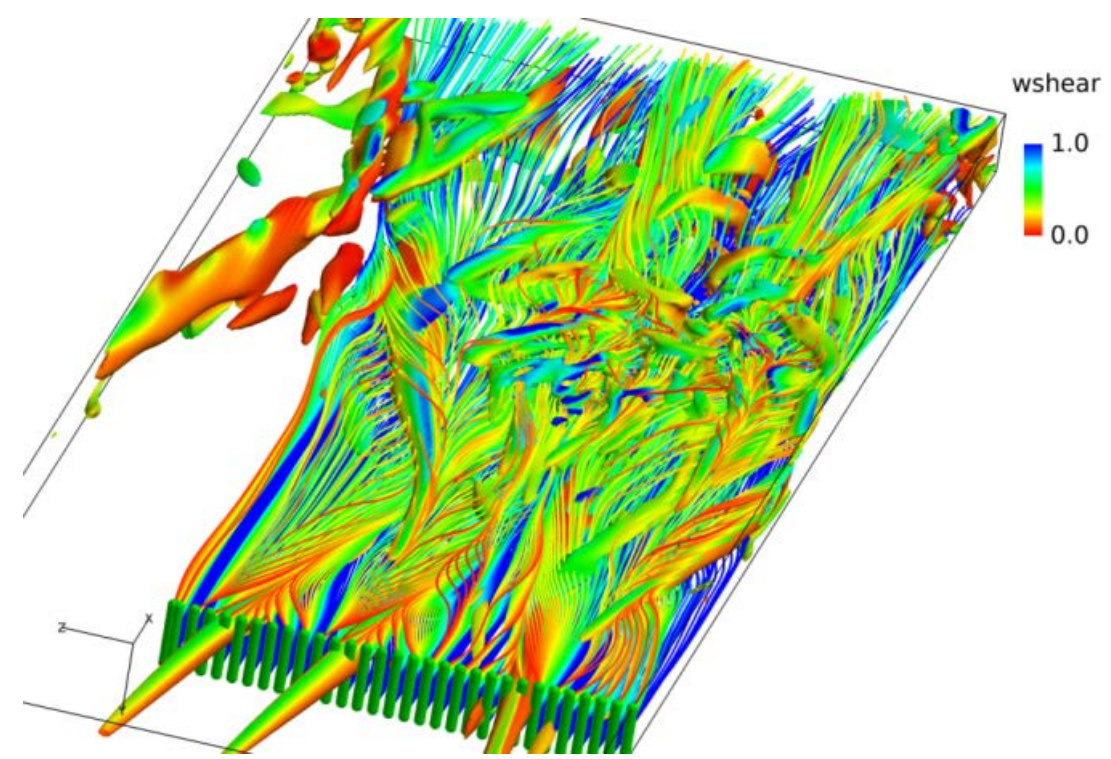

(b)

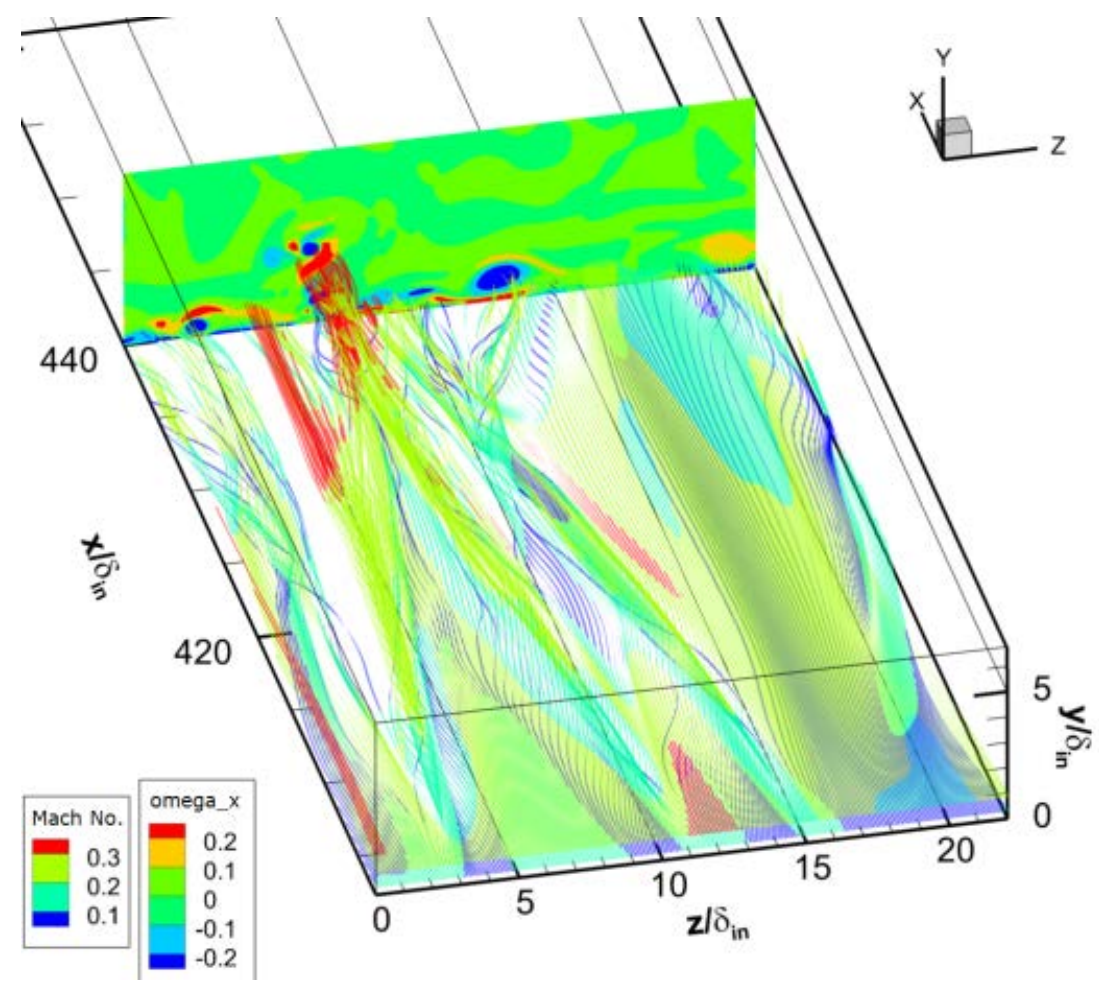

(c)

Figure 4 Continued. 


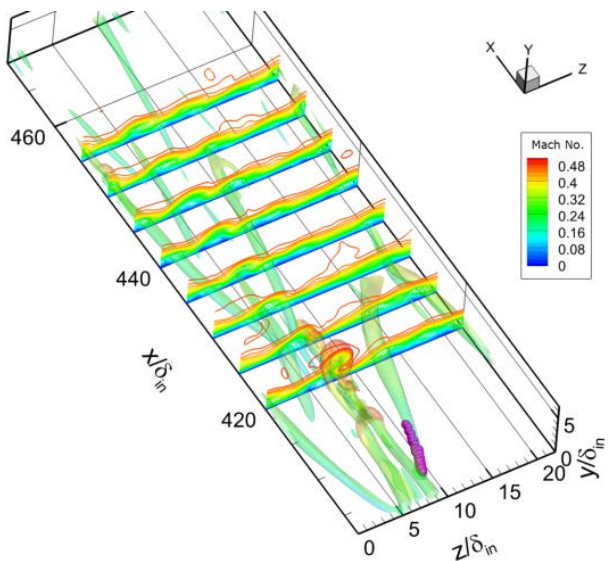

(a) $t^{*}=258.0 \delta_{\text {in }} / u_{\infty}$.

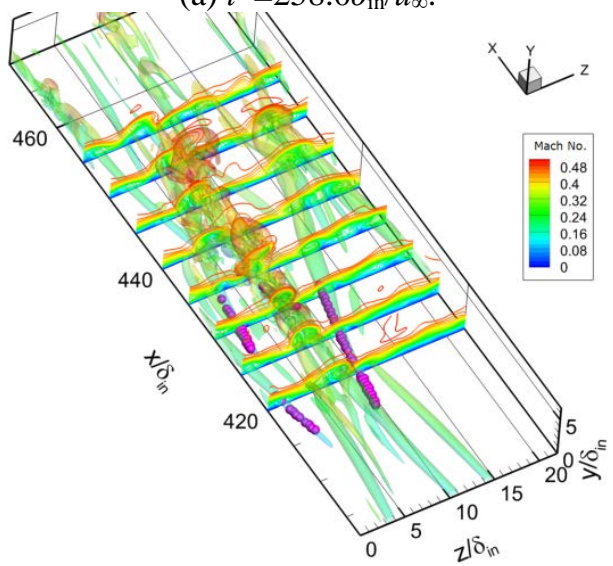

(c) $t^{*}=289.3 \delta_{\text {in }} / u_{\infty}$.

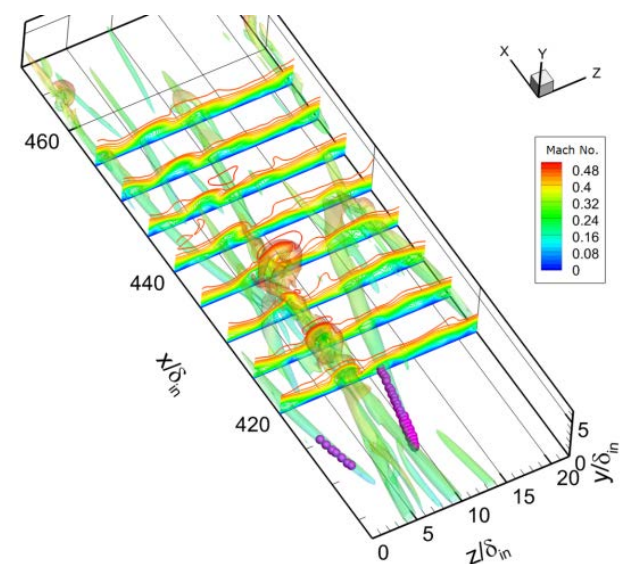

(b) $t^{*}=273.7 \delta_{\text {in }} / u_{\infty}$.

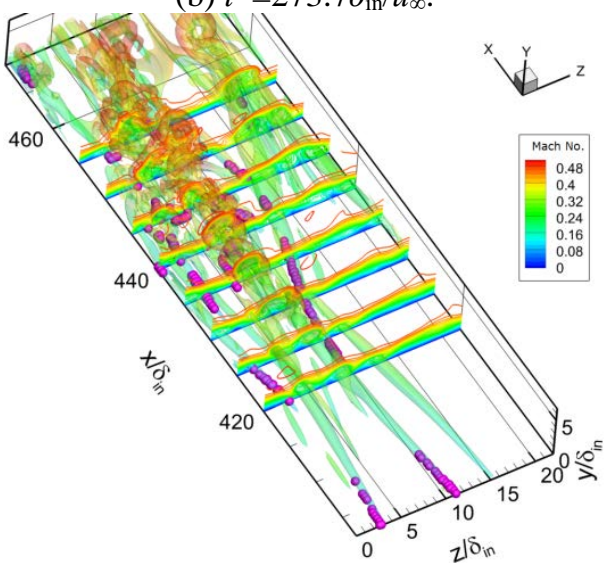

(d) $t^{*}=305.0 \delta_{\text {in }} / u_{\infty}$.

Figure 5: Time evolution of relationship between hairpin vortices, regions of high friction and low-speed streaks. Hairpin vortices are visualized by the partially transparent iso-surface of the velocity gradient tensor, $Q^{*}=4 \times 10^{-3}$. Here, the value is non-dimensionalized by $u_{\infty}$ and $\delta_{\text {in }}$. The color on the iso-surfaces is the streamwise velocity. Streamwise velocity contours are visualized at $x / \delta_{\text {in }}=420$ 455. The regions of high friction are visualized by pink particles.

\section{CONCLUSIONS}

The effects of FST on the evolution of hairpin vortices during the boundary-layer transition were investigated by DNS. FST intensities of $\mathrm{Tu}=0-6 \%$ were considered with a free-stream Mach number of 0.5 . The relationship between the high-friction regions at the wall and hairpin vortices was investigated by analyzing the computed flow fields. When FST is introduced along with inlet sinuous disturbances, asymmetric hairpin vortices and numerous secondary hairpin vortices, which are qualitatively different from the case of no FST, are generated. The arrangement of hairpin vortices also becomes different. High-friction regions are generated by vortices induced very close to the wall. The interior points of the near-wall vortices responsible for generating the high-friction regions can be clearly visualized as the group of points in $\mathbf{R}^{3}$. The newly derived judgement algorithm for interior points based on 
the Euler angle worked successfully. The high-friction regions are the tips of hairpin legs and the regions of near-wall vortices induced beneath the hairpin packet. These regions often correspond to the periphery of the hairpin packets. FST changes the distribution of high friction regions.

\section{ACKNOWLEDGEMENTS}

The present study is financially supported by IHI Corporation under a collaborative research project between Ehime University, Japan, and IHI Corporation. Computations were partly conducted using a supercomputer system at the Japan Aerospace Exploration Agency (JAXA-JSS2). This work was supported by a Grant for Basic Science Research Projects from the Sumitomo Foundation and the Institute of Statistical Mathematics (ISM) Cooperative Research Program 2016 ISM-CRP2019. Computational resources were provided by ISM and JAXA. We thank Stuart Jenkinson, PhD, from Edanz Group (www.edanzediting.com/ac) for editing a draft of this manuscript.

\section{REFERENCES}

[1] Smith, C.R., Walker, J.D.A., Haidari, A.H. \& Sobrun, U., On the dynamics of nearwall turbulence. Philos. Trans. R. Soc. London, Ser. A, 336, pp. 131-175, 1991.

[2] Theodorsen, T., Mechanism of turbulence. Proceedings of the 2nd Midwestern Conference on Fluid Mech. Bull., Ohio State University, Columbus, OH, 1952.

[3] Lee, C.B. \& Wu, J.Z., Transition in wall-bounded flows. Applied Mechanics Reviews, 61, 030802-1-21, 2008.

[4] Robinson, S.K., Coherent motions in the turbulent boundary layer. Ann. Rev. Fluid Mech., 23(601), pp. 601-639, 1991.

[5] Robinson, S.K., The kinematics of turbulent boundary layer structure. NASA TM 103859, 1991.

[6] Panton, R., Overview of the self-sustaining mechanisms of wall turbulence. Prog. Aero. Sci., 37, pp. 341-383, 2001.

[7] Adrian, R.J., Hairpin vortex organization in wall turbulence. Phys. Fluids, 19, 041301, 2007.

[8] Dennis, D.J.C., Coherent structures in wall-bounded turbulence. Ann. of the Brazilian Acad. Sci., 87(2), pp. 1161-1193, 2015.

[9] Moin, P., Leonard, A. \& Kim, J., Evolution of a curved vortex filament into a vortex ring. Phys. Fluids, 29(4), pp. 955-963, 1986.

[10] Acarlar, M. \& Smith, C., A study of hairpin vortices in a laminar boundary layer. Part I. Hairpin vortices generated by a hemisphere protuberance. J. Fluid Mech., 175, pp. $1-41$.

[11] Hon, T.L. \& Walker, J.D.A., Evolution of hairpin vortices in a shear flow. Computers and Fluids, 20(3), pp. 343-358, 1991.

[12] Singer, B.A. \& Joslin, R.D., Metamorphosis of a hairpin vortex into a young turbulent spot. Phys. Fluids, 6(11), pp. 3724-3736, 1994.

[13] Zhou, J., Adrian, R.J. \& Balachander, S., Autogeneration of near-wall vortical structures in channel flow. Phys. Fluids, 8(1), pp. 288-290, 1996.

[14] Zhou, J., Adrian, R.J. \& Balachandar, S., Mechanisms for generating coherent packets of hairpin vortices in channel flow. J. Fluid Mech., 387, pp. 353-396, 1999.

[15] Liu, C. \& Chen, L., Parallel DNS for vortex structure of late stages of flow transition. Computers \& Fluids, 45, pp. 129-137, 2011.

[16] Duguet, Y., Schlatter, P., Henningson, D. \& Eckhardt, B., Self-sustained localized structures in a boundary-layer flow. Phys. Rev. Lett., 108, 044501, 2012. 
[17] Cohen, J., Karp, M. \& Mehta, V., A minimal flow-elements model for the generation of packets of hairpin vortices in shear flows. J. Fluid Mech., 747, pp. 30-43, 2014.

[18] Eitel-Amor, G., Örlu, R., Schlatter, P. \& Flores, O., Hairpin vortices in turbulent boundary layers. Phys. Fluids, 27, 025108, 2015.

[19] Sabatino, D.R. \& Maharjan, R., Characterizing the formation and regeneration of hairpin vortices in a laminar boundary layer. Phys. Fluids., 27, 124104, 2015.

[20] Kim, K., Sung, H.J., Adrian, R.J., Effects of background noise on generating coherent packets of hairpin vorticies. Phys. Fluids., 20, 105107, pp. 1-10, 2008.

[21] Matsuura, K. \& Kato, C., Large-eddy simulation of compressible transitional flows in a low-pressure turbine cascade. AIAA Journal, 45(2), pp. 442-457, 2007.

[22] Matsuura, K. \& Nakano, M., A throttling mechanism sustaining a hole tone feedback system at very low Mach numbers. J. Fluid Mech., 710, pp. 569-605, 2012.

[23] Cebechi, T. \& Smith, A.M.O., Analysis of Turbulent Boundary Layer, Academic Press: New York, 1974.

[24] Jiang, M., Machiraju, R. \& Thompson, D., Detection and visualization of vortices. The Visualization Handbook, eds C.D. Hansen \& C.R. Johnson, Academic Press, pp. 295309, 2005.

[25] Sujudi, D. \& Haimes, R., Identification of swirling flow in 3-D vector fields. AIAA95-1715-CP, 1995.

[26] Kida, S. \& Miura, H., Identification and analysis of vortical structures. Eur. J. Mech. B/Fluids, 17(4), pp. 471-488, 1998.

[27] Cousteix, J., Turbulence et Couches Limites. Cépadués-Éditions, Toulouse, 1989.

[28] Anderson, J.D. Jr., Hypersonic and High-Temperature Gas Dynamics, AIAA, 2006.

[29] Matsuura, K., Matsui, K. \& Tani, N., Effects of free-stream turbulence on the global pressure fluctuation of compressible transitional flows in a low-pressure turbine cascade. Int. J. Num. Meth. Heat and Fluid Flow, 28(5), pp. 1-17, in press.

[30] Matsuura, K., Direct numerical simulation of a straight vortex tube in a laminar boundary-layer flow. Int. J. Comp. Meth. and Exp. Meas., 4(4), pp. 474-483, 2016. 